\title{
What can we learn from blood granulocyte patterns in patients with asthma?
}

\author{
Kirsty Hambleton and lan D. Pavord \\ Affiliation: Respiratory Medicine Unit, Nuffield Dept of Medicine, University of Oxford, Oxford, UK. \\ Correspondence: lan D. Pavord, Respiratory Medicine Unit, Nuffield Dept of Medicine, NDM Research \\ Building, Old Road Campus, University of Oxford, Oxford, OX3 7FZ, UK. E-mail: ian.pavordAndm.ox.ac.uk
}

@ERSpublications

Blood eosinophil counts are a potentially informative biomarker in airway disease http://ow.ly/aOHt3032iz0

There has been an explosion in interest in the utility of the blood eosinophil count as a biomarker in patients with airway disease. It is a readily accessible measure, and studies have shown a consistent positive relationship between the blood eosinophil count and the risk of exacerbations [1-6], and the extent to which this can be reduced by inhaled corticosteroids (ICS) [3-5] and anti-interleukin-5 monoclonal antibodies [6-8]. Much of the work to date has been carried out in patients with severe asthma or has involved post hoc analysis of intervention studies of ICS in patients with moderate-to-severe chronic obstructive pulmonary disease (COPD). Much less is known about the relationship between blood eosinophil counts and outcomes in patients with less severe asthma, and there is almost no information available from longitudinal studies. The study by NADIF et al. [9] published in this issue of the European Respiratory Journal is therefore welcome, as it provides new and interesting information in both areas.

The results presented form part of the Epidemiological Study on the Genetics and Environment of Asthma (EGEA), and include a cross-sectional analysis of 232 patients with asthma and 242 symptomatic first-degree relatives of these patients. Longitudinal data over 12 years were available from 242 participants who were aged $>16$ years at the time of the first survey. Blood eosinophil levels were categorised as high if $\geqslant 250$ cells per $\mathrm{mm}^{3}$ and blood neutrophils if $\geqslant 5000$ cells per $\mathrm{mm}^{3}$. These pre-defined levels were based on previous data and represent the 75th percentile of the distribution of blood granulocyte counts. Relationships between blood granulocyte categories, asthma symptoms and asthma exacerbations were analysed. The authors assessed how robust these relationships were by assessing different cut-points, and by determining the relationship between asthma outcomes and consistency of inflammatory pattern. By and large, these additional analyses supported and strengthened their major conclusions.

Asthma was identified using a self-reported set of four recognised questions and/or medical record review but no up-to-date spirometry was performed. Symptom control was assessed using responses to recognised questions related to the Global initiative for Asthma definition [10]. The authors report "uncontrolled disease" in $10 \%$ and $13 \%$ of participants in the cross-sectional analysis and longitudinal analysis groups, respectively, and "partly controlled disease" in approximately a third of participants. The overall use of ICS was low in relation to the reported level of asthma control, with only $25.7 \%$ participants reporting regular ICS use within the past 12 months, a median daily dose of $250 \mu \mathrm{g}$ (range 50-500 $\mu \mathrm{g}$ ) per day and $60.3 \%$ of all participants taking no medication in the preceding 12 months. The population studied could therefore be characterised as mild by treatment requirement criteria although they were symptomatic and undertreated.

Four main blood inflammatory cell patterns were identified: paucigranulocytic (48.9\%), eosinophilic (31.6\%), neutrophilic (10.6\%) and mixed (8.9\%). These inflammatory groups were relatively stable over 12 years. How these patterns relate to airway inflammation is unclear as no direct airway measurements were made. On the basis of other studies $[11,12]$, it is reasonable to conclude that the eosinophilic group

Received: Aug 052016 | Accepted: Aug 052016

Conflict of interest: Disclosures can be found alongside this article at erj.ersjournals.com

Copyright OERS 2016 
is more likely to have eosinophilic airway inflammation but no such assumptions can be made in the neutrophilic group. Systemic factors such as obesity, recent infection, age and systemic inflammation, as well more airway-specific factors such as smoking and corticosteroid use, could have influenced this measure. The neutrophil-high population tended to be older, was more female predominant and more likely to be treated with ICS, contained a higher proportion of committed smokers, and was more likely to have reported a respiratory infection within the last 4 weeks, supporting a diverse range of causes for raised blood neutrophils. In the longitudinal analysis, this population had poor asthma control and a higher frequency of exacerbations even when the analysis was adjusted for chronic bronchitis, ICS use, eosinophil counts and smoking status. Persistent neutrophilia was particularly strongly associated with these outcomes. No associations were seen with decline in lung function, a finding that is at variance with the consistent demonstration of a cross-sectional [13] and longitudinal [14] relationship between sputum neutrophil counts and decline in lung function.

In contrast, in the eosinophilic group, no obvious relationship was seen with symptoms or exacerbations, and there was no meaningful change in these variables in patients who transitioned from high to low or low to high over time. Patients in this group did have greater airway hyperresponsiveness, a lower forced expiratory volume in $1 \mathrm{~s}$ at follow-up and higher serum IgE. These findings differ from those of the DREAM study [6], which showed a strong positive relationship between exacerbation frequency and blood eosinophil count in patients with severe eosinophilic asthma. In addition, two large, community-based, observational studies have shown compelling evidence of a "dose-response" relationship between the blood eosinophil count and the occurrence of asthma attacks [1,2]. One potential explanation is that ICS use obscured the relationship between exacerbation frequency and blood eosinophils in the EGEA population. It will be difficult to be sure as little can be deduced about the relationship between biomarkers and specific treatment responses in a noninterventional study such as this. A better perspective would be available from placebo-controlled trials and future studies should prospectively investigate outcomes by different blood eosinophil thresholds. Post hoc analysis of studies of key placebo-controlled trials might also be possible. Such analyses have been performed with several studies of ICS in patients with COPD [3-5] but not yet in patients with asthma.

The analysis by NADIF et al. [9] is important as it suggests that mechanisms driving symptom expression can be disassociated from more traditional asthma-related measures. It follows that therapeutic strategies might need to move on from "one size fits all" symptom-based strategies to a new approach based on analysis of the main drivers of morbidity and targeted, personalised management [15]. A key unanswered question is the extent to which blood granulocyte patterns identify "treatable traits". Although no relationship was seen between blood eosinophils and important longer-term outcomes, we believe that this measure remains the best prospect for an informative biomarker, particularly if supplemented with a more airway-specific measure such as exhaled nitric oxide. There is also the tantalising prospect that these measures can be used to identify the main drivers of type-2 high inflammation and individualise treatment approaches [16].

\section{References}

1 Price DB, Rigazio A, Campbell JD, et al. Blood eosinophil count and prospective annual asthma disease burden: a UK cohort study. Lancet Respir Med 2015; 3: 849-858.

2 Malinovschi A, Fonseca JA, Jacinto T, et al. Exhaled nitric oxide levels and blood eosinophil counts independently associate with wheeze and asthma events in National Health and Nutrition Examination Survey subjects. J Allergy Clin Immunol 2013; 132: 821-827.e1-5.

3 Pascoe S, Locantore N, Dransfield MT, et al. Blood eosinophil counts, exacerbations, and response to the addition of inhaled fluticasone furoate to vilanterol in patients with chronic obstructive pulmonary disease: a secondary analysis of data from two parallel randomised controlled trials. Lancet Respir Med 2015; 3: 435-442.

4 Pavord ID, Lettis S, Locantore N, et al. Blood eosinophils and inhaled corticosteroid/longacting $\beta$-2 agonist efficacy in COPD. Thorax 2016; 71: 118-125.

5 Siddiqui SH, Guasconi A, Vestbo J, et al. Blood eosinophils: a biomarker of response to extrafine beclomethasone/ formoterol in chronic obstructive pulmonary disease. Am J Respir Crit Care Med 2015; 192: 523-525.

6 Pavord ID, Korn S, Howarth P, et al. Mepolizumab for severe eosinophilic asthma (DREAM): a multicentre, double-blind, placebo-controlled trial. Lancet 2012; 380: 651-659.

7 Castro M, Wenzel SE, Bleecker ER, et al. Benralizumab, an anti-interleukin 5 receptor alpha monoclonal antibody, versus placebo for uncontrolled eosinophilic asthma: a phase $2 \mathrm{~b}$ randomised dose-ranging study. Lancet Respir Med 2014; 2: 879-890.

8 Castro M, Zangrilli J, Wechsler ME, et al. Reslizumab for inadequately controlled asthma with elevated blood eosinophil counts: results from two multicentre, parallel, double-blind, randomised, placebo-controlled, phase 3 trials. Lancet Respir Med 2015; 3: 355-366.

9 Nadif R, Siroux V, Boudier A, et al. Blood granulocyte patterns as predictors of asthma phenotypes in adults from the EGEA study. Eur Respir J 2016; 48: 1040-1051.

10 Reddel HK, Bateman ED, Becker A, et al. A summary of the new GINA strategy: a roadmap to asthma control. Eur Respir J 2015; 46: 622-39.

11 Schleich F, Corhay JL, Louis R. Blood eosinophil count to predict bronchial eosinophilic inflammation in COPD. Eur Respir J 2016; 47: 1562-1564. 
12 Wagener $\mathrm{AH}$, de Nijs $\mathrm{SB}$, Lutter R, et al. External validation of blood eosinophils, $\mathrm{FE}_{\mathrm{NO}}$ and serum periostin as surrogates for sputum eosinophils in asthma. Thorax 2015; 70: 115-120.

13 Shaw DE, Berry MA, Hargadon B, et al. Association between neutrophilic airway inflammation and airflow limitation in adults with asthma. Chest 2007; 132: 1871-1875.

14 Stanescu D, Sanna A, Veriter C, et al. Airways obstruction, chronic expectoration, and rapid decline of FEV1 in smokers are associated with increased levels of sputum neutrophils. Thorax 1996; 51: 267-271.

15 Agusti A, Bel E, Thomas M, et al. Treatable traits: toward precision medicine of chronic airway diseases. Eur Respir J 2016; 47: 410-419.

16 Pavord ID, Hilvering B. Biomarkers and inhaled corticosteroid responsiveness in asthmatic patients. J Allergy Clin Immunol 2015; 135: 884-885. 